\section{Langfristige Behandlung der primären pulmonalen Hypertonie mit inhalativem lloprost $^{1}$}

Zusammenfassung: Hintergrund: Die kontinuierliche Infusion von Prostacyclin ist eine effektive Behandlung der primären pulmonalen Hypertonie. Allerdings ist hierfür ein permanenter zentraler Venenzugang erforderlich mit dem entsprechenden Risiko infektiöser Komplikationen. In den letzten Jahren ist die Anwendung von inhalativem Iloprost, einem stabilen Prostacyclinderivat als alternative Behandlungsmöglichkeit für Patienten mit primärer pulmonaler Hypertonie beschrieben worden. Methodik: Wir untersuchten die Auswirkungen einer einjährigen Behandlung mit lloprost-Aerosol auf die körperliche Belastbarkeit und die Hämodynamik bei Patienten mit primärer pulmonaler Hypertonie. Ergebnisse: Vierundzwanzig Patienten mit primärer pulmonaler Hypertonie erhielten lloprost-Aerosol in einer kumulativen Tagesdosis zwischen 100 und $150 \mu \mathrm{g}$. Die mittlere $( \pm S D)$ Gehstrecke im 6-min-Gehtest stieg darunter von $278 \pm 96$ Metern vor Therapiebeginn auf $363 \pm 135$ Meter nach 12-monatiger Therapie an $(\mathrm{P}<0,0001)$. Während des gleichen Zeitraumes fiel der mittlere pulmonal-arterielle Druck von $59 \pm 10 \mathrm{mmHg}$ auf $52 \pm 15 \mathrm{mmHg}$ ab $(P=0,006)$, das Herzminutenvolumen stieg von $3,8 \pm 1,4 \mathrm{I} / \mathrm{min}$ auf $4,4 \pm 1,3 \mathrm{l} / \mathrm{min}$ $(P=0,02)$, und der pulmonal-vaskuläre Widerstand fiel von $1,205 \pm 467$ dynes $\cdot \mathrm{s} \cdot \mathrm{cm}^{-5}$ auf $925 \pm 469$ dynes $\cdot \mathrm{s} \cdot \mathrm{cm}^{-5}$ ab $(P=0,0003)$. Die Behandlung wurde allgemein gut vertragen und außer leichtem Husten sowie geringfügigen Kopf- und Kieferschmerzen wurden keine Nebenwirkungen beobachtet. Schlussfolgerung: Die langfristige Behandlung mit inhalativem Iloprost ist sicher und zeigt dauerhafte Effekte auf die körperliche Belastbarkeit und die Hämodynamik bei Patienten mit primärer pulmonaler Hypertonie.

Long-Term Treatment of Primary Pulmonary Hypertension with Aerosolized Iloprost, a Prostacyclin Analogue: Background: Continuous intravenous infusion of prostacyclin is an effective treatment for primary pulmonary hypertension. This approach, however, requires the insertion of a permanent central venous catheter with the potential risk of serious complications. Recently, administration of aerosolized iloprost, a stable prostacyclin analogue, has been introduced as an alternative therapy for severe pulmonary hypertension. Methods: We evaluated the effects of treatment with aerosolized iloprost over a one-year period on exercise capacity and hemodynamic variables in patients with primary pulmonary hypertension. Results: Twenty-four patients with primary pulmonary hyperten-

Pneumologie 2001; 55: 38-43

(c) Georg Thieme Verlag Stuttgart · New York ISSN 0934-8387
M. M. Hoeper, M. Schwarze, S. Ehlerding,

A. Adler-Schuermeyer, E. Spiekerkoetter,

J. Niedermeyer, M. Hamm, H. Fabel

Abteilung Pneumologie, Medizinische Hochschule Hannover sion received aerosolized iloprost at a cumulative daily dose of 100 to $150 \mu \mathrm{g}$ for at least one year. The mean ( \pm SD) walking distance in the 6-min-walk test increased from $278 \pm 96$ meters at base line to $363 \pm 135$ meters after 12 months $(P<0.0001)$. During the same period, the mean pulmonary artery pressure declined from $59 \pm 10 \mathrm{mmHg}$ to $52 \pm 15 \mathrm{mmHg}(P=0.006)$, the cardiac output increased from $3.8 \pm 1.4 \mathrm{I} / \mathrm{min}$ to $4.4 \pm 1.3 \mathrm{l} / \mathrm{min}$ $(P=0.02)$, and the pulmonary vascular resistance declined from $1,205 \pm 467$ dynes $\cdot \mathrm{s} \cdot \mathrm{cm}^{-5}$ to $925 \pm 469$ dynes $\cdot \mathrm{s} \cdot \mathrm{cm}^{-5}$ $(P=0.0003)$. Treatment was generally well tolerated and except for mild coughing, minor headache and jaw pain in some patients, no side effects occurred. Conclusions: Long-term treatment with aerosolized iloprost is safe and has sustained effects on exercise capacity and pulmonary hemodynamics in patients with primary pulmonary hypertension.

\section{Einleitung}

Es konnte in verschiedenen Studien gezeigt werden, dass die Behandlung der primären pulmonalen Hypertonie mit kontinuierlich intravenös appliziertem Prostacyclin (Epoprostenol) die körperliche Belastbarkeit, hämodynamische Parameter und das Überleben verbessert [1-7]. Allerdings hat diese Form der Behandlung auch gravierende Nachteile. Zum einen die Toleranzentwicklung, die bei einigen Patienten eine kontinuierliche Dosissteigerung erforderlich macht und dadurch zu den hohen Therapiekosten beiträgt. Das andere, klinisch relevantere Problem ist direkt mit dem zentralvenösen Zugang und dem Verabreichungssystem bedingt und besteht in dem Risiko schwer wiegender Infektionen, Katheterthrombosen und Pumpenversagen. Derartige Komplikationen werden zwar als selten beschrieben, sind aber potenziell lebensbedrohlich [4,7].

Die Nachteile der intravenösen Prostacyclintherapie könnten durch die kürzlich von Olschewski et al. beschriebene inhalative Applikation von Iloprost [8], einem stabilen Prostacyclinanalogon kompensiert werden $[9,10]$. Inhalatives Iloprost ist ein potenter pulmonaler Vasodilatator, der bei Patienten mit primärer pulmonaler Hypertonie wirksamere hämodyamische Akuteffekte aufweist als inhalatives Stickstoffmonoxid [11]. Außerdem gibt es erste Beobachtungen, dass die inter-

\footnotetext{
${ }^{1}$ Deutsche Fassung von: Long-term treatment of primary pulmonary hypertension with aerosolized iloprost, a prostacyclin analogue N. Engl. J. Med. 342; (2000): 1866-1870

Zitat nur nach dieser Originalquelle
} 
mittierende Inhalation von Iloprost günstige Auswirkungen bei Patienten mit primärer und sekundärer pulmonaler Hypertonie hat [11,12]. Allerdings gibt es bislang kaum Berichte über den langfristigen Therapieeffekt von IloprostAerosol bei Patienten mit primärer pulmonaler Hypertonie [9].

Wir berichten über die Auswirkungen einer einjährigen Behandlung mit aerosolisiertem Iloprost bei Patienten mit primärer pulmonaler Hypertonie.

\section{Methodik}

\section{Patienten}

Wir beschreiben den klinischen Verlauf der Patienten, die uns zwischen März 1997 und Juni 1998 zur Behandlung einer pulmonalen Hypertonie zugewiesen wurden und bei denen eine primäre pulmonale Hypertonie entsprechend den Kriterien der National Institutes of Health diagnostiziert wurde [13]. Patienten mit sekundärer pulmonaler Hypertonie wurden ebenso ausgeschlossen wie Patienten mit schwerem Rechtsherzversagen, die bei der Erstvorstellung bereits mit Katecholaminen behandelt wurden.

In Deutschland ist weder intravenöses Prostacyclin noch inhalatives Iloprost für die Behandlung der pulmonalen Hypertonie zugelassen. Wir bieten in unserem Zentrum der Mehrzahl der Patienten mit fortgeschrittener primärer pulmonaler Hypertonie zunächst eine Behandlung mit IloprostAerosol auf Heilversuchsbasis an. Unsere Ethikkommission hat diesem Vorgehen zugestimmt und alle Patienten werden über den experimentellen Charakter dieser Therapie schriftlich aufgeklärt.

Für die Behandlung mit inhalativem Iloprost mussten die Patienten eine schwere Beeinträchtigung ihrer körperlichen Leistungsfähigkeit aufweisen (New York Heart Association Klasse III oder IV), die auf konventionelle Therapie inklusive Kalziumantagonisten nicht ansprach. Die körperliche Leistungsfähigkeit wurde mit dem 6-Minuten-Gehtest quantifiziert, nachdem die Patienten sich in zwei Probeläufen mit dem Test vertraut gemacht hatten [14].

Die Hämodynamik wurde im Rahmen einer Rechtsherzkatheteruntersuchung bestimmt. Das Herzminutenvolumen wurde mittels der Thermodilutionsmethode gemessen [15]. Nach Dokumentation der Ausgangswerte wurde die akute hämodynamische Reaktion auf Iloprost-Aerosol ermittelt. Dazu wurden $50 \mu \mathrm{g}$ Iloprost (Ilomedin ${ }^{\circledR}$, Schering, Berlin) in $5 \mathrm{ml}$ isotoner Kochsalzlösung verdünnt und mit einem Jet-Vernebler (Ilo-Neb ${ }^{\mathrm{TM}}$; Fa. Nebu-Tec, Elsenfeld, Deutschland) aerolisiert. Auf diese Weise wurden während einer 10-15-minütigen Inhalation zwischen 14 und $17 \mathrm{~g}$ Iloprost appliziert. Direkt im Anschluss an die Inhalation sowie alle 15 Minuten während der folgenden Stunde wurden alle hämodynamischen Parameter dokumentiert, um den Maximaleffekt sowie den zeitlichen Verlauf der Wirkung festzuhalten.

Alle Patienten begannen die Behandlung mit einer täglichen Iloprost-Dosis von $100 \mu \mathrm{g}$ aufgeteilt in 6-8 Inhalationen (alle 2-3 Stunden ohne Unterbrechung der Nachtruhe). Nachdem pharmakokinetische Untersuchungen von Iloprost-Aerosol nicht verfügbar sind, orientierte sich das Dosierungsschema an den ersten Berichten von Olschewski et al [9]. Inhalationsdosis und Zeitintervall richteten sich nach den Informationen, die während der Akuttestung gewonnen wurden. Wenn der pulmonal-vaskuläre Widerstand während dieser Testung um mehr als 20 Prozent abfiel, aber innerhalb von einer Stunde wieder auf die Ausgangswerte anstieg, inhalierten die Patienten achtmal pro Tag. Alle anderen Patienten begannen mit 6 Inhalationen pro Tag.

Die Patienten wurden aus der stationären Behandlung entlassen, nachdem sie sich mit der Therapie und dem Equipment vertraut gemacht hatten und wurden danach alle 4-12 Wochen zu ambulanten Kontrollen einbestellt. Nach 3 und 12 Monaten wurden die Patienten erneut stationär aufgenommen, um die 6-Minuten-Gehteste und die Rechtsherzkatheteruntersuchungen zu wiederholen. Die Gehteste wurden alle auf der gleichen Strecke durchgeführt. Die Katheteruntersuchungen begannen am frühen Morgen vor der ersten IloprostInhalation, also nach einer 10-12-stündigen Inhalationspause und wurden nach dem gleichen Protokoll wie die Ausgangsuntersuchungen durchgeführt.

\section{Statistik}

Alle hämodynamischen Parameter und die Ergebnisse der 6Minuten-Gehteste werden als Mittelwert \pm SD dargestellt. Die hämodynamischen Akuteffekte von Iloprost wurden mit dem gepaartem t-Test ausgewertet. Die Veränderungen der 6Minuten-Gehteste und der Hämodynamik zwischen Ausgangszeitpunkt und nach 3 bzw. 12 Monaten wurden mittels Varianzanalyse für wiederholte Messungen verglichen. Wenn dieser Globaltest signifikante Unterschiede anzeigte, wurde ein gepaarter t-Test angeschlossen, um Unterschiede zwischen den einzelnen Zeitpunkten herauszufinden. Bei Patienten, die nicht in der Lage waren, den Gehtest durchzuführen, wurde die Gehstrecke mit 0 Metern angesetzt. Es wurden jeweils nur die hämodynamischen Daten verglichen, die vor Iloprost-Inhalation erhoben wurden (Präinhalationswerte). Die lineare Regressionsanalyse wurde benutzt, um die Akuteffekte von Iloprost-Aerosol zum Ausgangszeitpunkt mit den Präinhalationswerten nach 12-monatiger Therapie zu vergleichen [16]. Alle statistischen Teste wurden 2-seitig durchgeführt. Ein p-Wert < 0,05 galt als Signifikanzgrenze.

\section{Ergebnisse}

Zwischen März 1997 und Juni 1998 wurde 31 Patienten mit primärer pulmonaler Hypertonie mit Iloprost-Aerosol behandelt. Sieben dieser Patienten wurden von der Auswertung ausgeschlossen, weil sie an anderen Zentren weiter betreut wurden. Keiner dieser Patienten starb während des Beobachtungszeitraums. Die übrigen 24 Patienten wurden über mindestens ein Jahr mit Iloprost-Aerosol behandelt und erhielten vollständige Herzkatheteruntersuchungen und Belastungstests. Fünfzehn der Patienten waren Frauen, neun Männer. Das mittlere Alter betrug $38 \pm 12$ Jahre (22 bis 65 Jahre). Wie aus Tab. 1 ersichtlich, litten alle Patienten an einer schweren pulmonalen Hypertonie, befanden sich also in einer funktionellen New York Heart Association Klasse III $(n=20)$ oder IV $(n=4)$. Die initiale Tagesdosis von Iloprost betrug bei allen Patienten zunächst $100 \mu \mathrm{g}$. Bei sechs Patienten wurde diese Dosis im Verlauf auf $150 \mu \mathrm{g}$ erhöht, weil nach dreimonatiger 
Tab. 1 Hämodynamik und Ergebnisse des 6-Minuten-Gehtests bei 24 Patienten mit primärer pulmonaler Hypertonie unter inhalativem lloprost*

\begin{tabular}{|c|c|c|c|c|c|c|}
\hline & \multicolumn{2}{|c|}{ Ausgangszeitpunkt } & \multicolumn{2}{|c|}{ nach 3 Monaten } & \multicolumn{2}{|c|}{ nach 12 Monaten } \\
\hline & vor & nach & vor & nach & vor & nach \\
\hline Herzfrequenz (Schläge/min) & $84 \pm 13$ & $84 \pm 13$ & $82 \pm 15$ & $79 \pm 13$ & $82 \pm 10$ & $80 \pm 10$ \\
\hline mittlerer systemischer Blutdruck $(\mathrm{mmHg})$ & $98 \pm 14$ & $100 \pm 14$ & $93 \pm 10 \dagger$ & $92 \pm 12$ & $90 \pm 13 \dagger$ & $89 \pm 13$ \\
\hline mittlerer pulmonal-arterieller Druck $(\mathrm{mmHg})$ & $59 \pm 10$ & $50 \pm 13$ & $52 \pm 11 \dagger$ & $44 \pm 12$ & $52 \pm 15 \dagger$ & $43 \pm 16$ \\
\hline rechtsatrialer Druck $(\mathrm{mmHg})$ & $8 \pm 7$ & $7 \pm 6$ & $5 \pm 4 \dagger$ & $4 \pm 4$ & $5 \pm 4 \dagger$ & $4 \pm 4$ \\
\hline Herzminutenvolumen (I/min) & $3,8 \pm 1,4$ & $4,5 \pm 1,4$ & $4,0 \pm 1,2$ & $4,5 \pm 1,2$ & $4,4 \pm 1,3 \dagger$ & $4,8 \pm 1,4$ \\
\hline pulmonal-vaskulärer Widerstand (dynes $\cdot \mathrm{s} \cdot \mathrm{cm}^{-5}$ ) & $1205 \pm 467$ & $866 \pm 415$ & $1001 \pm 437 \dagger$ & $728 \pm 330$ & $925 \pm 469 \dagger$ & $704 \pm 440$ \\
\hline systemischer Widerstand (dynes $\cdot \mathrm{s} \cdot \mathrm{cm}^{-5}$ ) & $2088 \pm 712$ & $1791 \pm 508$ & $1884 \pm 506 \dagger$ & $1646 \pm 397$ & $1660 \pm 494 \dagger+$ & $1534 \pm 467$ \\
\hline Schlagvolumen (ml/Schlag) & $46 \pm 16$ & $55 \pm 16$ & $50 \pm 16 \dagger$ & $57 \pm 15$ & $55 \pm 16 \dagger \ddagger$ & $61 \pm 19$ \\
\hline gemischt-venöse $\mathrm{O}_{2}$-Sättigung (\%) & $62 \pm 8$ & $68 \pm 8$ & $65 \pm 7 \dagger$ & $70 \pm 7$ & $67 \pm 8 \dagger$ & $70 \pm 7$ \\
\hline 6-Minuten-Gehtest (Meter) & $278 \pm 96$ & n.d. & $353 \pm 69 \dagger$ & n.d. & $363 \pm 135 \dagger$ & n.d. \\
\hline
\end{tabular}

* Angaben in Mittelwert \pm SD; vor und nach geben die Werte vor und direkt nach Inhalation von lloprost an; n. d. bedeutet nicht durchgeführt $\dagger \mathrm{p}<0,05$ für den Vergleich der Präinhalationswerte nach 3 und 12 Monaten mit den Ausgangswerten

$\pm p<0,05$ für den Vergleich der Präinhalationswerte nach 3 und 12 Monaten

Therapie keine Zunahme der körperlichen Belastbarkeit nachzuweisen war. Bei den anderen Patienten blieb die Dosis über das gesamte Jahr konstant. Alle Patienten wurden antikoaguliert. Diuretika wurden nach klinischem Bedarf gegeben. Eine Begleitmedikation mit Digitalis oder Kalziumantagonisten wurde nicht geändert.

Wie aus Tab. 1 ersichtlich, bestätigte der 6-Minuten Gehtest die schwere Leistungseinschränkung der Patienten zum Ausgangszeitpunkt. Nach dreimonatiger Behandlung mit Iloprost-Aerosol nahm die Gehstrecke um $75 \pm 67$ Meter zu ( $P<0,0001)$. Dieser Effekt war bei der 12-Monats-Kontrolle weiterhin nachweisbar, auch wenn kein weiterer Anstieg der Belastbarkeit gegenüber der 3-Monats-Kontrolle nachweisbar war.

Unter der initialen Kathetertestung kam es nach Inhalation von Iloprost zu einem Abfall des pulmonal-arteriellen Druckes um 8,9 $\pm 9,2$ mmHg (im Mittel - 15 Prozent; $\mathrm{P}<0,0001$ ), begleitet von einem Anstieg des Herzminutenvolumens um $0,7 \pm 0,5 \mathrm{l} / \mathrm{min}$ (im Mittel +20 Prozent; $\mathrm{P}<0,0001$ ) und einer Reduktion des pulmonal-vaskulären Widerstands um $339 \pm 260$ dynes $\cdot \mathrm{s} \cdot \mathrm{cm}^{-5}$ (im Mittel - 28 Prozent; $\mathrm{P}<0,0001$ ) . Herzfrequenz und systemischer Blutdruck blieben unbeeinflusst. Während der Herzkatheteruntersuchungen nach 3und 12-monatiger Behandlung konnte eine praktisch identische Reaktion auf Iloprost dokumentiert werden (Tab.1).

Verglichen mit den Ausgangswerten zeigte sich nach 3monatiger Behandlung mit inhalativem Iloprost eine signifikante Verbesserung der Präinhalationswerte für den pulmonal-arteriellen Druck, den pulmonal-vaskulären Widerstand, das Schlagvolumen, die gemischt-venöse Sättigung und den rechtsatrialen Druck (Tab.1). Diese Effekte waren auch nach 12-monatiger Therapie weiter nachweisbar. Außerdem zeigte sich bei der 12-Monats-Kontrolle ein signifikanter Anstieg des Herzminutenvolumens entsprechend einer langfristigen Verbesserung der rechtsventrikulären Funktion unter Langzeittherapie mit inhalativem Iloprost. Ein Vergleich der hämodynamischen Parameter zum Ausgangszeitpunkt und nach 12 Monaten (vor Inhalation gemessen) zeigte eine Abnahme des pulmonal-arteriellen Druckes um 6,5 $\pm 8,7 \mathrm{mmHg}$ (im Mittel - 12 Prozent; $\mathrm{P}=0,006$ ), eine Reduktion des pulmonal-vaskulären Widerstands um $280 \pm 323$ dynes $\cdot \mathrm{s} \cdot \mathrm{cm}^{-5}$ (im Mittel 23 Prozent; $\mathrm{P}=0,0003$ ) und eine Abnahme des rechtsatrialen Druckes um $3 \pm 4 \mathrm{mmHg}(\mathrm{P}=0,01)$. Außerdem stieg das Herzminutenvolumen um $0,6 \pm 1,3 \mathrm{l} / \mathrm{min}$ an (im Mittel +22 Prozent; $P=0,02$ ), die Herzfrequenz blieb konstant, das Schlagvolumen stieg um $9 \pm 16 \mathrm{ml}$ (im Mittel + 28 Prozent; $\mathrm{P}=0,009)$ und die gemischt-venöse Sauerstoffsättigung stieg um $5 \pm 8$ Prozent (mittlerer relativer Anstieg +8 Prozent; $\mathrm{P}=0,01$ ) (Tab. 1). Bei 13 der 24 Patienten (54 Prozent) war nach 12 Monaten ein Abfall des pulmonal-vaskulären Widerstands um mindestens 20 Prozent nachweisbar (Abb.1). Zu jedem Untersuchungszeitpunkt konnte eine weitere Verbesserung der Hämodynamik unmittelbar nach Iloprost-Inhalation dokumentiert werden (Tab.1).

Um zu überprüfen, ob der Akuttest mit Iloprost eine Aussage über den langfristigen Therapieerfolg zulässt, verglichen wir die Akutveränderungen der Hämodynamik im Rahmen der ersten Katheteruntersuchung mit den Veränderungen der Präinhalationswerte nach 12-monatiger Therapie (Abb.2). Dabei zeigte sich, dass Patienten mit einer stärkeren Akutreaktion üblicherweise auch langfristig stärker von der Therapie profitierten $(r=0,66, P<0,01)$. Dennoch konnte auch bei 7 von 12 Patienten, bei denen der pulmonal-vaskuläre Widerstand im Rahmen der Akuttestung um weniger als 20 Prozent abfiel, im Langzeitverlauf eine anhaltende Verbesserung nachgewiesen werden, während bei einem Patienten keine Änderung der Hämodynamik nachweisbar war und es bei 4 Patienten sogar zu einer Verschlechterung des pulmonal-vaskulären Widerstands kam (Abb. 2). Zwei der zuletzt genannten Patienten wurden im weiteren Verlauf auf kontinuierlich intravenös verabreichtes Iloprost umgestellt, allerdings ohne klinische Verbesserung, so dass eine Aufnahme auf die Lungentransplantationswarteliste erfolgte.

Die Behandlung mit inhalativem Iloprost wurde von allen Patienten gut vertragen. Husten trat bei den meisten Patienten während der ersten Behandlungstage auf, ließ aber im weiteren Verlauf nach und trat nach 4 Wochen praktisch nicht 


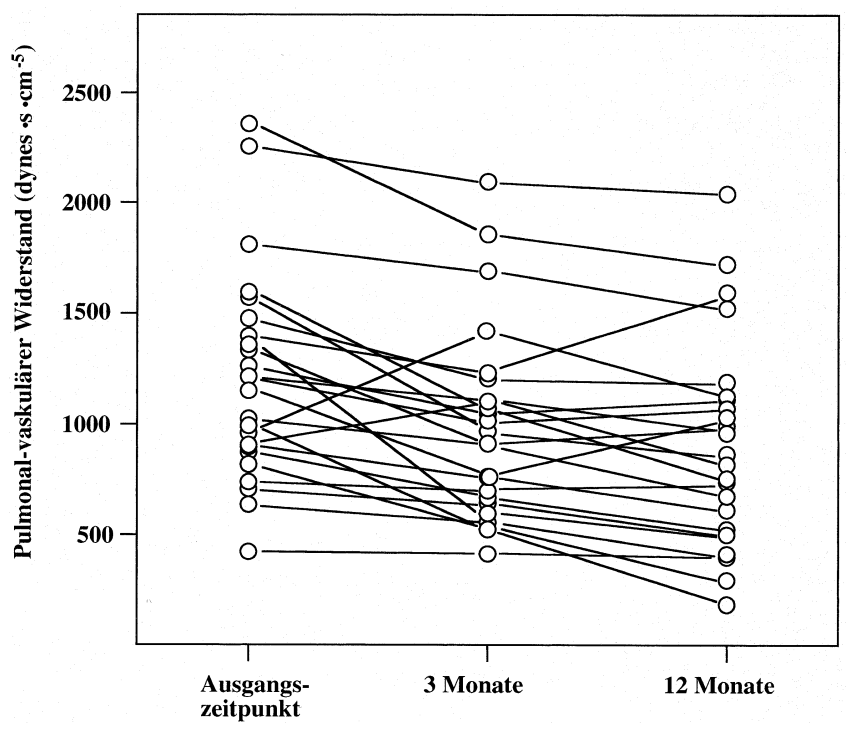

Abb. 1 Pulmonal-vaskulärer Widerstand zum Ausgangszeitpunkt sowie nach 3- und 12-monatiger Therapie mit aerosolisiertem lloprost.

Der pulmonal-vaskuläre Widerstand nahm bei allen bis auf zwei Patienten nach 3-monatiger Therapie ab, gefolgt von einer weiteren Abnahme nach 12 Monaten in allen bis auf sechs Patienten. Verglichen mit dem Ausgangszeitpunkt fand sich bei 19 von 24 Patienten nach 12 Monaten ein reduzierter pulmonal-vaskulärer Widerstand.

mehr auf. Die Lungenfunktionsdaten blieben über den gesamten Beobachtungszeitraum stabil ebenso wie Blutbild, Elektrolyte, Retentionswerte und Leberfunktionsteste (Daten nicht gezeigt). Fünf Patienten berichteten über Gesichtsröte, Kopfschmerzen und Kiefergelenksschmerzen gegen Ende der Inhalationen, aber all diese Nebenwirkungen wurden als leicht beschrieben und eine spezielle Behandlung bzw. ein Absetzen der Medikation war nicht erforderlich. Keiner der Patienten klagte über gastrointestinale Beschwerden. Die Herzkatheteruntersuchungen zeigten zwar eine signifikante Abnahme von arteriellem Blutdruck und systemischem Widerstand, aber eine symptomatische Hypotension trat bei keinem unserer Patienten auf. Darüber hinaus kam es nicht zu Problemen wie Schwindel oder Synkopen, die Ausdruck von Rebound-Phänomenen während der Inhalationspausen hätten sein können. Trotzdem beschrieben nahezu alle Patienten Schwankungen in ihrer körperlichen Belastbarkeit, die typischerweise unmittelbar nach Inhalation als am besten empfunden wurde, um dann über die nächsten 2-3 Stunden bis zur nächsten Inhalation kontinuierlich abzunehmen.

\section{Diskussion}

Unsere Ergebnisse zeigen, dass die langfristige Behandlung von Patienten mit primärer pulmonaler Hypertonie zu einer Verbesserung der körperlichen Belastbarkeit und der hämodynamischen Parameter führt.

Die therapeutische Wirksamkeit der kontinuierlichen Behandlung der primären pulmonalen Hypertonie mit kontinuierlich intravenös appliziertem Prostacyclin ist inzwischen allgemein akzeptiert, aber die Mechanismen, über die Prostaglandine den Krankheitsverlauf beeinflussen, werden bis heute nicht verstanden. Verschiedene Studien haben deutlich

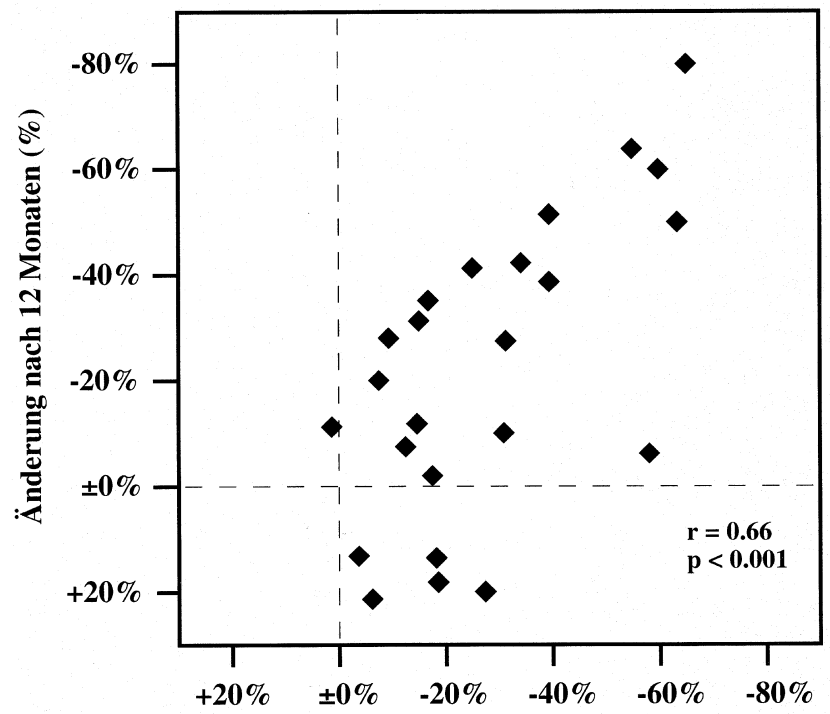

Kurzfristige Änderung zum Ausgangszeitpunkt (\%)

Abb. 2 Regressionsanalyse der Akuteffekte von inhalativem lloprost auf den pulmonal-vaskulären Widerstand zum Ausgangszeitpunkt und der Veränderungen der Präinhalationswerte des pulmonal-vaskulären Widerstands nach 12-monatiger Behandlung mit inhalativem lloprost. Im Allgemeinen zeigten Patienten mit der stärksten Akutantwort auf inhalatives lloprost auch die größten Therapieeffekte nach 12monatiger Behandlung. Allerdings konnte auch bei 7 von 12 Patienten mit nur geringem Akutansprechen nach 12-monatiger Behandlung ein nennenswerter Therapieerfolg dokumentiert werden.

gemacht, dass die Langzeiteffekte von Prostacyclin bei pulmonaler Hypertonie über eine reine Vasodilatation hinausgehen. Darüber hinaus sind wahrscheinlich auch antithrombotische und antiproliferative Wirkungen sowie bislang unzureichend charakterisierte Effekte auf Wachstumsfaktoren und das vaskuläre Remodeling für die langfristigen Wirkungen mitverantwortlich [4, 7,8,17 - 19]. Einige dieser Effekte wie beispielsweise die Plättchenaggregationshemmung könnten die Plasmahalbwertzeit von Prostaglandinen überdauern. Es wurde bislang noch nicht untersucht, ob Prostaglandine wirklich kontinuierlich appliziert werden müssen, um ihr therapeutisches Potenzial bei pulmonaler Hypertonie entfalten zu können. Diese Frage ist allerdings von zentraler Bedeutung, wenn es um die Anwendung von aerosolisiertem Iloprost als alternative Behandlungsmode für diese Patienten geht. Auch wenn die Plasma-Halbwertzeit von Iloprost mit 20-30 min angegeben wird [8], sind die akuten hämodynamischen Effekte einer einzelnen Inhalation nach einer Stunde praktisch nicht mehr nachweisbar [11]. Daher ist unsere Beobachtung, dass sich auch die Präinhalationswerte (die nach einer nächtlichen Inhalationspause ermittelt wurden) nach einer einjährigen Behandlung mit aerosolisiertem Iloprost verbesserten, als Hinweis dafür zu werten, dass tatsächlich andere Mechanismen als eine reine Vasodilatation zu der therapeutischen Wirkung beigetragen haben. Die Beobachtungen, dass die Akutantwort auf die Inhalation von Iloprost auch nach einem Jahr noch erhalten war, und dass die Abnahme des pulmonal-vaskulären Widerstands nach 12 Monaten die Wirkung zum Ausgangszeitpunkt überstieg, unterstützen diese Schlussfolgerung. 
Inhalatives Iloprost kann auf nichtinvasivem Wege verabreicht werden, was als potenzieller Vorteil gegenüber intravenösem Prostacyclin, das über einen zentralvenösen Zugang appliziert werden muss, angesehen werden kann. Die entscheidende Frage ist allerdings, ob inhalatives Iloprost genauso effektiv wie intravenöses Prostacyclin die Mortalität von Patienten mit primärer pulmonaler Hypertonie senkt und deren körperliche Belastbarkeit verbessert.

Obgleich intravenöses Prostacyclin seit über 10 Jahren für die Behandlung der pulmonalen Hypertonie eingesetzt wird, gibt es erstaunlich wenigen Daten über den langfristigen Effekt dieser Behandlung. Erst kürzlich haben McLaughlin et al. ihre Erfahrungen mit langfristiger intravenöser Prostacyclinbehandlung bei primärer pulmonaler Hypertonie publiziert [7]. In dieser Studie hatte die Anwendung von intravenösem Adenosin im Akutversuch vergleichbare Effekte wie inhalatives Iloprost bei unseren Patienten. McLaughlin et al. berichteten über eine Abnahme des pulmonal-vaskulären Widerstands um 53 Prozent nach 12- bis 24-monatiger Behandlung mit intravenösem Iloprost. Dieser Wert übertrifft die Abnahme des pulmonal-vaskulären Widerstands um 23 Prozent, die wir bei unseren Patienten nach einjähriger Behandlung mit Iloprost-Aerosol beobachtet haben. Allerdings konnten wir nach einem Jahr unmittelbar nach Inhalation von Iloprost eine Abnahme des pulmonal-vaskulären Widerstands um 42 Prozent gegenüber den Ausgangswerten vor Therapiebeginn beobachten und dieser Wert ist mit den Resultaten der kontinuierlichen intravenösen Prostacyclinbehandlung wie von McLaughlin et al. beschrieben durchaus vergleichbar [7].

Die Dosis von aerosolisiertem Iloprost betrug bei der Mehrzahl unserer Patienten während des gesamten Beobachtungszeitraumes $100 \mu \mathrm{g} / \mathrm{Tag}$ und musste bei sechs Patienten auf $150 \mu \mathrm{g} /$ Tag gesteigert werden, weil deren Ansprechen auf die Behandlung als unzureichend bewertet wurde. Wir wissen allerdings nicht, ob dieses Dosisschema tatsächlich optimal war, oder ob mit höheren Dosierungen, anderen Inhalationsintervallen oder einer regelmäßigen Dosiseskalation bessere klinische Resultate erzielt worden wären. In der Studie von McLaughlin et al. wurde die Dosis des intravenösen Prostacyclin monatlich im Mittel um 2,4 ng/ $\mathrm{kg} / \mathrm{min}$ bis $\mathrm{zu}$ einer mittleren Dosis von $40 \mathrm{ng} / \mathrm{kg} / \mathrm{min}$ gesteigert [7]. In Deutschland würde die Behandlung eines Patienten bei diesen Dosierungen mehr als DM 500.000,- pro Jahr kosten. Die jährlichen Behandlungskosten mit aerosolisiertem Iloprost liegen mit dem in dieser Studie angewendetem Verneblersystem bei einer täglichen Dosis von 100-150 $\mu \mathrm{g}$ bei etwa DM 150.000,-. Mit neu entwickelten Ultraschallverneblern lassen sich diese Kosten um nahezu 50 Prozent reduzieren [20].

Inhalatives Iloprost wurde grundsätzlich gut vertragen und kein Patient brach die Behandlung wegen unerwünschter Begleiterscheinungen ab. Das Auftreten von Kieferschmerzen und Gesichtsröte bei einigen Patienten sowie der signifikante, wenn auch klinisch asymptomatische Blutdruckabfall zeigen, dass inhalatives Iloprost kein selektiver pulmonaler Vasodilatator ist, sondern dass es zu einem Übertritt der Substanz in die systemische Zirkulation kommt. Es gibt weiterhin Unsicherheiten bezüglich der Sicherheit der Behandlung mit inhalativem Iloprost. Dazu gehört die Möglichkeit des Auftretens von Reboundphänomenen zwischen den Inhalationen, insbesondere während der nächtlichen Inhalationspause. Wir konnten bei unseren Patienten allerdings keine klinischen oder hämodynamischen Hinweise für das Auftreten solcher Reboundphänome feststellen. Andererseits sind Reboundphänomene eine bekannte und gefürchtete Komplikation der kontinuierlichen intravenösen Prostacyclintherapie, wenn es zu abrupten Unterbrechungen der Medikamentenzufuhr kommt [21]. Allerdings beschrieben nahezu alle unserer Patienten Schwankungen ihrer körperlichen Leistungsfähigkeit zwischen den Inhalationen. Diese Fluktuationen wurden von unseren Patienten gut toleriert, könnten aber bei Patienten mit fortgeschrittener Rechtsherzinsuffizienz nachteilige Folgen haben. Außerdem zeigten 5 unserer 24 Patienten (21 Prozent) offenbar keine Reaktion auf die Behandlung mit inhalativem Iloprost und es ist unklar, ob solche Patienten von einer weiteren Dosiseskalation oder von einer Umstellung auf intravenöses Prostacyclin profitieren würden.

Alles in allem erlauben unsere Beobachtungen den Schluss, dass aerosolisiertes Iloprost eine effektive Behandlung der primären pulmonalen Hypertonie darstellt. Gegenwärtig laufen in Europa Phase-III-Studien, von denen die Zulassung von inhalativem Iloprost für die Behandlung der pulmonalen Hypertonie erwartet wird. Mit diesem neuen Therapieansatz nehmen die therapeutischen Möglichkeiten für die fortgeschrittene pulmonale Hypertonie zu, aber auch die Unsicherheiten über die optimale Behandlung dieser Patienten. Damit ist die Zeit gekommen, Studien durchzuführen, die Langzeiteffekte von intravenösem Prostacyclin und inhalativem Iloprost direkt vergleichen.

\section{Literatur}

${ }^{1}$ Higenbottam TW, Wheeldon D, Wells F, Wallwork J. Long-term treatment of primary pulmonary hypertension with continuous intravenous epoprostenol (prostacyclin). Lancet 1984; I: 10461047

${ }^{2}$ Rubin LJ, Mendoza J, Hood M et al. Treatment of pulmonary hypertension with infusion of prostacyclin (epoprostenol). Ann Intern Med 1990; 12: 485-491

${ }^{3}$ Barst RJ, Rubin LJ, McGoon MD et al. Survival in primary pulmonary hypertension with long-term continuous intravenous prostacyclin. Ann Intern Med 1994; 121: 409-415

${ }^{4}$ Barst RJ, Rubin LJ, Long WA et al. A comparison of continuous intravenous epoprostenol (prostacyclin) with conventional therapy for primary pulmonary hypertension. N Engl J Med 1996; 334: $296-301$

${ }^{5}$ Shapiro SM, Oudiz RJ, Cao T et al. Primary pulmonary hypertension: Improved long-term effects and survival with continuous intravenous epoprostenol infusion. J Am Coll Cardiol 1997; 30: $343-349$

${ }^{6}$ Hinderliter AL, Willis PW, Barst RJ et al. Effect of long-term infusion of prostacyclin (epoprostenol) on echocardiographic measures of right ventricular structure and function in primary pulmonary hypertension. Circulation 1997; 95: 1479-1486

${ }^{7}$ McLaughlin VV, Genthner D, Panella M, Rich S. Reduction in pulmonary vascular resistance with long-term epoprostenol (prostacyclin) therapy in primary pulmonary hypertension. $\mathrm{N}$ Engl J Med 1998; 338: 272 - 277

${ }^{8}$ Grant SM, Goa KL. Iloprost. A review of its pharmakodynamic properties, and therapeutic potential in peripheral vascular disease, myocardial ischemia and extracorporeal circulation procedures. Drugs 1992; 43: 889-924 
${ }^{9}$ Olschewski H, Walmrath D, Schermuly R et al. Aerosolized prostacyclin and iloprost in severe pulmonary hypertension. Ann Intern Med 1996; 124: 820-824

10 Olschewski H, Ghofrani HA, Schmehl T et al. Inhaled iloprost to treat severe pulmonary hypertension: an uncontrolled trial. Ann Intern Med 2000; 132: 435-443

${ }^{11}$ Hoeper MM, Olschewski H, Ghofrani HA et al. A comparison of the acute hemodynamic effects of inhaled nitric oxide and aerosolized iloprost in primary pulmonary hypertension. J Am Coll Cardiol 2000; 35: 176-182

12 Olschweski H, Ghofrani HA, Walmrath D et al. Inhaled prostacyclin and iloprost in severe pulmonary hypertension secondary to lung fibrosis. Am J Respir Crit Care Med 1999; 160: 600-607

${ }^{13}$ Rich S, Dantzker DR, Ayres SM et al. Primary pulmonary hypertension: a national prospective study. Ann Intern Med 1987; 107: $216-223$

${ }^{14}$ Guyatt GH, Sullivan MJ, Thompson PJ et al. The 6-minute walk: a new measure of exercise capacity in patients with chronic heart failure. Can Med Assoc J 1985; 132: 919-923

${ }^{15}$ Hoeper MM, Maier R, Tongers J et al. Determination of cardiac output by Fick's method, thermodilution, and acetylene rebreathing in pulmonary hypertension. Am J Resp Crit Care Med 1999; 160: 535-541

${ }^{16}$ Rosner B. Fundamentals of biostatistics 4th ed. Belmont, CA: Duxbury Press, 1995

${ }^{17}$ Hoeper MM, Voelkel NF, Bates TO, Allard JD, Horan M, Shepherd D, Tuder RM. Prostaglandins induce vascular endothelial growth factor in a human monocygotic cell line and in rat lungs via cAMP. Am J Respir Cell Mol Biol 1997; 17: 748-756

${ }^{18}$ Fishman AP. Pulmonary hypertension - beyond vasodilator therapy. N Engl J Med 1998; 338: 321 - 322

${ }^{19}$ Fishman AP. Epoprostenol (prostacyclin) and pulmonary hypertension. Ann Intern Med 2000; 132: 500-550

${ }^{20}$ Gessler T, Schmehl T, Seeger W. Comparison of two inhalation systems for Ilomedin-aerosol therapy in pulmonary hypertension. Am J Respir Crit Care Med 1999; 159: A159 (abstract)

${ }^{21}$ Rubin LJ. Primary pulmonary hypertension. N Engl J Med 1997; 336: $111-117$
Priv.-Doz. Dr. med. M. M. Hoeper

Medizinische Hochschule Hannover

Abteilung Pneumologie

30623 Hannover

E-mail: KMHoeper@AOL.com 